\title{
Amerika
}

Mémoires, identités, territoires

12 | 2015 :

La mort : imaginaires et sociétés

Thématique

L'imaginaire de la mort en littérature

\section{L'imaginaire transatlantique de la mort : rites, mythes et conflits}

\author{
NiKOL DZIUB
}

\section{Résumés}

Français English

Washington Irving rapporte de ses voyages en Europe des images de la mort et de la vie après la mort, il s'imprègne de l'imaginaire romantique du cimetière et se confronte aux interrogations nouvelles sur la succession et l'héritage. Mais il s'intéresse aussi aux civilisations disparues sans laisser de traces, aux grands conflits destructeurs, à l'anéantissement des cultures traditionnelles par l'industrialisation. Nous interrogeons également les rituels funéraires des aborigènes d'Amérique et la perception qu'en ont les Occidentaux. Nous mettons notamment la figure du dernier de la race en relation avec l'imaginaire mythique de l'éternel retour, du Jugement dernier et du Déluge. Il se construit un imaginaire américain de la mort à la fois apocalyptique et puritain, fondé sur les interrelations entre Natives et Européens. La mort est entourée de rituels qui expriment et construisent les valeurs culturelles nationales. La circulation transatlantique des mythes et des rites funéraires mène à une stratification interculturelle qui fonctionne aussi bien individuellement que globalement.

From his journeys in Europe, Washington Irving brings back images of death and of life after death. He absorbs the romantic imaginary of cemeteries and he is faced with new questions about inheritance. But he is also interested in ancient civilizations and in major destructive conflicts, in order to depict the annihilation of traditional cultures by industrialization. The paper also examines the European perception of American aborigines' funeral rites. We argue that the figure of the last of the race is in relation with the myth of the eternal return, of the Last Judgment and with the flood myth. American representations of death are both apocalyptic and puritanical, and are based on the interrelations between Natives and Europeans. Death is surrounded by rituals which express and build the national cultural values. The transatlantic circulation of funeral myths and rites leads to a cross-cultural stratification which works as well at the level of the individual as on a global level. 
Mots-clés : mythe, représentations de la violence, identité nationale, mythes et pratiques funéraires, circulations transatlantiques

Keywords : myth, to represent violence, national identity, burial myths and practices, transatlantic circulations

Géographique : Amérique du Nord, Amérique hispanique, Andalousie

\section{Texte intégral}

L'imaginaire de la mort en Amérique se construit sur le fonds biblique. La mer (comme en général les eaux) est parmi les éléments essentiels du topos métaphorique du Jugement dernier. Elle rend « les morts qui étaient ensevelis dans ses eaux », et à ce phénomène succède une seconde mort : " l'enfer et la mort furent jetés dans l'étang de feu » (Apocalypse XX, 13-14). Il se développe ainsi une vision apocalyptique du monde, qui suppose qu'il y a une vie après la mort, et c'est ce mythe que la littérature réinvestit pour tenter de répondre aux questions de la modernité. Mais en même temps, la terreur de la mort et de la catastrophe cède progressivement le pas à la lumière du Nouveau monde. L'idée d'une révélation finale est contenue en germe dans le mot grec apocalypsis. Le cataclysme, la crise et le catastrophe précèdent l'âge d'or sur la terre nouvelle. " Après cela je vis un ciel nouveau et une terre nouvelle » (Apocalypse XXI, 1).

Justement, en Europe, le XIXe siècle s'ouvre sur les Révolutions et l'abolition des monarchies, et c'est avec la fin de l'ère des princes que naissent les nations ${ }^{1}$. Et, grâce aux voyages et au contact intense des hommes de lettres américains avec l'Europe, le fonds imaginaire européen s'introduit dans la culture américaine. La littérature américaine naissante se veut indépendante des lettres anglaises, et, pour être à la fois originale et originelle, elle cherche à retrouver ses fondateurs dans les reliques de son histoire précolombienne. La Création de l'identité d'une nation passe par l'identification de ses ancêtres ${ }^{2}$ : aux États-Unis, c'est Washington Irving qui s'est chargé dans une large mesure de cette tâche.

Écrivain américain, mais littérairement formé en Angleterre, Washington Irving, dans sa quête des origines de l'Amérique, s'intéresse à l'histoire de l'Europe, et notamment à celle de l'Espagne. Par ailleurs, il admet l'action de Dieu dans l'histoire, la fatalité lui semblant décisive dans les grandes crises. Pour l'écrivain romantique, cela suppose qu'il est possible, narrativement parlant, de représenter le lien entre le fini et l'infini. Le thème prophétique et apocalyptique permet de mettre en rapport l'histoire et la fiction, de doter les personnages principaux d'un sur-pouvoir et d'inscrire les événements dans une totalité. Le Romantisme américain parvient ainsi à se distinguer des romantismes européens, en ceci que la vie éternelle si recherchée y est occultée par la mort omniprésente.

\section{La fin des Dynasties et la naissance de New-York}

4 La carrière littéraire de Washington Irving commence avec The History of New York, signée du pseudonyme de Diedrich Knickerbocker. Le sens de l'humour, du wit, et l'approche originale de l'écriture de l'histoire qui s'y révèlent attirent l'attention de Walter Scott. Mais le ton moqueur de l'œuvre ne fait que masquer un malheur profond, l'auteur semble tenter de cacher combien le passé pèse sur le présent. Le récit commence pourtant $a b$ ovo, Irving retrace avec une piquante ironie l'histoire de ce fragment du monde depuis la naissance de l'univers jusqu'à la fin de la dynastie danoise. Le mythe de l'Exode vient ici soutenir l'idéal démocratique. Irving se sert du sarcasme pour attaquer les oppresseurs que sont pour lui les rois 
européens, et pour défendre les opprimés - les Américains. Son propos est d'immortaliser par la littérature les civilisations disparues. Dans son prélude adressé au public, il cite Aristote :

"Wars, conflagrations, deluges," says Aristotle, "destroy nations, and with them all their monuments, their discoveries, and their vanities. - The torch of science has more than once been extinguished and rekindled - a few individuals, who have escaped by accident, reunite the thread of generations." 3

C'est dans ce texte que le motif du torrent, de l'eau destructrice aussi bien que féconde apparaît. Parmi les possibles « beginnings of the world », Irving cite la théorie de Hutton, qui dit que les eaux sont la source de tout, même si elles ont en même temps le pouvoir et la puissance de mettre fin au monde, qui pourrait être " washed away by the force of rain, rivers, and mountain torrents, until it is confounded with the ocean, or, in other words, absolutely dissolves into itself ${ }^{4}$. Or, la prise de possession des terres est fréquemment représentée comme un raz-de-marée chez Irving. Cette image de la mer et de son déplacement a deux significations : l'une liée au baptême (mais aussi à la conversion forcée) et l'autre à la fuite par les eaux vers un monde meilleur.

\section{La traversée de l'Océan}

Washington Irving voit son pays natal plein d'une jeunesse prometteuse, mais il lui manque ces trésors accumulés avec l'âge que sont les ruines de l'Europe - où chaque pierre suscite des associations poétiques, et où chaque tombe est d'une grandeur ténébreuse où résonne l'écho du passé. L’Océan qui sépare le Monde ancien du Nouveau Monde est donc en quelque sorte libérateur, puisqu'il permet de retrouver la wilderness ${ }^{5}$. Irving fait cependant la traversée dans le sens inverse. Arrivé en Europe, le voyageur s'engage dans une forme d'errance, qui lui permet de découvrir les mœurs des différents pays qu'il visite et de rendre compte des scènes de la vie locale dont il est témoin. Tel est le motif de la fugue de l'Américain en Europe, et le propos du Sketch-Book of Geoffrey Crayon (1819), tel qu'il est annoncé dans la Préface. L'épigraphe, tiré de The Anatomy of melancholy de Robert Burton ( « I have no wife nor children, good or bad, to provide for. ») prépare le lecteur à des sketches qui mêleront l'humour et la mélancolie. Mais surtout, cette citation révèle que la mort est d'abord pour Irving le moment où se transmet un héritage. En l'absence de successeurs, les richesses littéraires et culturelles meurent, les livres et leurs auteurs tombent dans l'oubli. Si l'écrivain peut revivre grâce aux lecteurs de son livre, les livres oubliés font partie du topos romantique qui entoure l'artiste incompris ou maudit : la bibliothèque est " a kind of literary catacomb, where authors, like mummies, are piously entombed, and left to slacken and molder in dusty oblivion $»^{6}$. Le Gothic américain est une réfraction de l'anglais : toutefois, là où le second évoque directement le passé pour remédier à ses vices, le premier superpose le présent et le passé, et forge ainsi l'image d'un Ancien Monde historique mais vague et mythologisé7. Cette esthétique de l'entre-deux (temporel et géographique) intervient dans la représentation du spirituel comme dans celle du matériel : dans le Sketch-Book, les mœurs et les traditions de l'Europe d'une part et de l'Amérique d'autre part sont ainsi comparées les unes aux autres.

Les traits de caractère et les habitudes sont en lien direct avec l'environnement. Dans le sketch intitulé Traits of Indian Character, Irving écrit que l'Indien d'Amérique du Nord « is formed for the wilderness, as the Arab is for the desert $»^{8}$. Défenseur des aborigènes, Irving rappelle qu'ils ont été " dispossessed of their hereditary possessions ${ }^{9}$ et que les écrivains ont dénaturé leurs mœurs et leur histoire. Irving met en valeur le courage des Indiens face à la mort : c'est là un des points essentiels par lesquels l'Indien se différencie de l'Européen. 


\section{Comment surmonter la mort ?}

C'est une question qui oriente les observations de Washington Irving, quand il note avec admiration que " No hero of ancient or modern days can surpass the Indian in his lofty contempt of death, and the fortitude with which he sustains its cruelest affliction $»^{10}$. L'héroïsme de celui qui affronte la mort est proportionnel à la cruauté de celui qui le tue. L'Européen " rushes to glorious death at the cannon's mouth », tandis que l'Indien « calmly contemplates its approach » : son détachement à l'égard de la mort matérielle " behold him rising superior to the white man ${ }^{11}$. Le lecteur suit l'auteur et ses personnages dans leur méditation devant la mort, et il ressent à son tour ce $"$ sublime and holy feeling ${ }^{12}$.

The Indians are remarkable for the reverence which they entertain for the sepulchers of their kindred. Tribes that have passed generations exiled from the abodes of their ancestors, when by chance they have been travelling in the vicinity, have been known to turn aside from the highway, and, guided by wonderfully accurate tradition, have crossed the country for miles to some tumulus, buried perhaps in woods, where the bones of their tribe were anciently deposited ; and there have passed hours in silent meditation. ${ }^{13}$

Quoique se réfugier dans ses pensées pour méditer soit une posture typiquement romantique, Irving admire avec sincérité le sang-froid des Indiens, qui n'exposent pas leurs émotions, mais les gardent celées dans leur âme. Il répand aussi l'idée selon laquelle l'Indien croit en une forme de métempsychose : la mort, pour l'Indien, n'est pas la fin de la vie, elle n'est pas définitive et doit être silencieuse, il invoque ses pères pour qu'ils constatent qu'il meurt sans gémissements. Mais quoiqu'il en soit de sa pensée eschatologique, l'Indien ne cédera jamais devant l'ennemi. Même le rabaissement physique contribue à lui faire honneur ${ }^{14}$. L'idée que l'Indien va jusqu'à exciter ses ennemis à inventer de nouvelles tortures se rapproche de ce qu'on lit dans les Notes sur la Virginie de Jefferson, où il est dit qu'il n'existe pas un seul exemple d'un «Indien tombé au pouvoir de ses ennemis qui ait demandé la vie ». Tocqueville aussi se réfère à cette phrase, quand il décrit un prisonnier indien qui cherche " la mort des mains de ses vainqueurs, en les insultant et en les provoquant de toutes les manières $»^{15}$.

Pour autant, Irving ne se désintéresse pas des pratiques mortuaires européennes. En méditant sur les lieux délaissés de l'Ancienne Europe, il étudie les mœurs et les rituels funéraires. Dans Westminster Abbey, il examine ce qui entretient le souvenir des morts, et il déplore que les sépultures baignent dans une atmosphère malsaine.

But why should we thus seek to clothe death with unnecessary terrors, and to spread horrors round the tomb of those we love? The grave should be surrounded by everything that might inspire tenderness and veneration for the dead ; or that might win the living to virtue. It is the place, not of disgust and dismay, but of sorrow and meditation. ${ }^{16}$

Dans un geste partiellement métadiscursif, l'auteur s'interroge sur les effets des représentations de la mort, sur ce qu'éprouve le lecteur quand on le confronte, dans une fiction, à la terreur. Irving rappelle que les images liées à la mort ne font pas toujours peur, mais suscitent aussi une forme de plaisir chez le spectateur. Un topos gothique encadre ainsi la réflexion de l'écrivain, qui pense que la puissance du silence est purificatrice. Irving reprend en fait les idées puritaines de Cotton Mather sur la fatalité du destin et l'imprévisibilité de la mort, mais surtout il puise dans ses récits mystiques. La légende de Sleepy Hollow serait entre autres inspirée des récits providentiels du théoricien du puritanisme. Le personnage principal, Ichabod, sait par cœur les « Cotton Mather's History of New-England Witchcraft, in which, by the way, he most firmly and potently believed ${ }^{17}$. Irving superpose à la Magnalia de Mather des événements merveilleux supposément advenus dans le Connecticut et le 
récit d'apparitions terribles, qui lui ont appris (et qui ont appris à ses personnages) comment échapper à la mort.

\section{La mort dans la Vieille Espagne, entre histoire et fiction}

Après l'Angleterre, la patrie littéraire d'Irving, c'est l'Espagne, qui l'intéresse dans la mesure où il pense y retrouver ses origines. Invité par Alexander Everett à traduire les Voyages of Columbus, Irving résiste à la facilité et décide d'écrire un récit fondé sur ses recherches personnelles in situ - ce qui le mènera à écrire parallèlement The Life and Voyages of Columbus et The Conquest of Granada. Le premier ouvrage est publié en 1828. Irving retrouve alors pleinement sa popularité, et en 1829, il publie $A$ Chronicle of the Conquest of Granada. Ce sont ses recherches qui le conduisent à séjourner en Andalousie et à vivre à l'Alhambra. Mais le Sud de l'Espagne lui évoque aussi des souvenirs d'enfance, de son voyage sur les bords du Hudson, et plus largement du passé non-corrompu de l'Amérique ${ }^{18}$.

Dans les couloirs de l'Alhambra, Irving replonge dans l'angoisse et tente de surmonter sa propre terreur : après la mort précoce de sa fiancée, il apprivoise la Mort à la manière des Indiens, en s'enfermant dans le silence de la douleur ${ }^{19}$. La Mort inquisitoriale et l'ensevelissement prématuré attirent son attention. Dans The Alhambra (1832), il raconte l'histoire d'une jeune fiancée qu'on enterre vivante : le mariage lui semble le contraire de la wilderness, contracter cette union indissoluble jusqu'à la mort, c'est pour lui être enfermé dans une tombe vivante. Irving opère un glissement hypallagique, et la femme enterrée vivante prête sa vie à la tombe qui l'a engloutie, elle est condamnée à mourir dans une « living tomb $»^{20}$. L'auteur décrit la pâleur mortelle de la fiancée, que tous (et surtout son père, qui la regarde " unmoved, without a tear ${ }^{21}$ ) considèrent comme morte, car les rites qui accompagnent la mort sont plus suggestifs que la mort elle-même.

\section{La légende du roi Rodéric}

Mais Irving ne se limite pas à ce qui lui est personnel : il donne à sa réflexion une dimension à la fois politique et cosmique. Il décrit par exemple la mort inavouée du Maure, dont le corps est d'abord amené hors de la ville, avant d'être brûlé sur les bords du Génil ${ }^{22}$. Cette cérémonie escamotée rappelle les textes de Cotton Mather, qui raconte comment les Indiens cachaient la dépouille de leurs congénères christianisés sous l'eau, en creusant un trou dans la glace, afin que personne ne retrouve leur corps ${ }^{23}$.

Mais surtout, c'est au dernier des survivants de relier les ères et les mondes, c'est à lui que revient le tâche messianique de fermer un cycle cosmique ${ }^{24}$ et d'en ouvrir un nouveau - celui de l'« âge d'or » qui naît de la mort d'un monde. The Legend of Don Roderick fait partie des Legends of the Conquest of Spain et elle est entrée dans les Spanish Voyages of Discovery et dans les Spanish Papers d'Irving. La mer et la mort se lient dans le mythe de l'Al-Andalus - Rodéric est vaincu au terme de la bataille de Gaudalete, et le fleuve est surnommé The River of the Death. Irving explique la défaite de l'armée des Goths devant celle des Musulmans par l'union dans la diversité dont ont fait preuve ces derniers ${ }^{25}$. Le gouvernement des Goths intéresse l'écrivain américain, puisque s'y opposent la monarchie élective et la monarchie héréditaire. La pureté du sang des Goths est également primordiale, mais en même temps l'union des nations conquérantes lui parait exemplaire : cette communauté est à ses yeux la meilleure manière d'agir pour attaquer l'ennemi ou pour défendre son territoire. Et 
c'est parce qu'elles rassemblent les nations que les troupes conquérantes peuvent être comparées à un raz-de-marée.

16 En effet, la légende, déjà réinvestie par les poètes britanniques (tels Walter $\mathrm{Scott}^{26}$ et Robert Southey, qui, dans le contexte de la Guerre d'Indépendance espagnole, réinterprètent l'histoire du roi Rodéric, le dernier des Goths), est utilisée pour défendre l'idée d'un esprit national et pour souligner l'importance de l'unification des peuples européens. La fin du règne goth est aussi celle du gouvernement monarchique, elle signifie donc qu'une ère nouvelle commence. Dans Roderick : the Last of the Goths de Robert Southey, la mort est présente dès l'incipit ${ }^{27}$ - ce poème est une élégie qui pleure la mort du dernier roi. Ce dernier porte le poids moral de la mort d'une race dont il est le représentant et l'héritier : il lui échoit d'être le dernier et de souffrir pour toute sa famille (« The unhappy chance which, rescuing him from death./ Left him the last of all his family $»^{28}$ ). Pour décrire l'invasion mauresque, Southey fait appel à l'imaginaire biblique : les envahisseurs sont arrivés en Espagne par le sud comme « a cloud of locusts $»^{29}$, comme un nuage de sauterelles. L'invasion détruit une culture, mais il reste un survivant ( $«$ the living death $»^{30}$ ), et cette dernière citadelle de chair qui lutte non plus pour elle-même mais pour tous les autres poétise la mort de la race.

\section{Le raz-de-marée de l'invasion}

Irving déplore ainsi que chaque renouvellement politique s'accompagne de la perte d'une race ou d'une culture. Et c'est précisément dans la quête des fragments du passé que réside pour lui le prix du travail de l'historien.

Le raz-de-marée (ou le torrent, ou la tempête) devient l'une des métaphores favorites du romancier, qui parle du « storm of Moslem invasion ${ }^{31}$, ou encore du " torrent of Moslem invasion » ${ }^{32}$. Il s'agit là bien sûr d'une métonymie, puisque les conquérants traversent la Méditerranée. Mais surtout, le déluge efface le passé et donne naissance à l'avenir. La distance entre la vie et la mort, comme celle qui sépare l'Andalousie de l'Amérique, est " like a golden sea ${ }^{33}$. Irving reprend, développe et file la métaphore mythifiante du raz-de-marée, qui est mobilisée une fois de plus pour évoquer l'arrivée des Maures en Andalousie. Leur puissance sans bornes, leur violence et leur hégémonie sont comparables à une mer déchaînée : «A remote wave of the great Arabian inundation, cast upon the shores of Europe, they seem to have all the impetus of the first rush of the torrent $»^{34}$. Le torrent est une image de la foule mouvante, c'est une métaphore du mouvement migratoire et conquérant, et des échanges essentiels à la vie américaine. Certains lieux, c'est vrai, dans les profondeurs du monde et au centre du continent, restent clos aux flux, comme une vallée dormante (A Sleepy Hollow), ou comme l'eau qui stagne. C'est que, si la tempête annonce la mort d'une race, l'accalmie, qui est l'« image même de l'univers de la Mort, qui est le commencement et la fin de la tempête, [...], et qui rend toute chose dont elle s'empare, insupportablement lourde ", la parachève ${ }^{35}$. Pourtant, l'invasion est d'abord une tempête déchaînée qui efface une race et transforme le monde : l'eau, la mer et l'océan servent de cloisons entre les civilisations, et on ne viole ces frontières que par désir de mourir ou de tuer.

19 La fin du règne maure et de la Reconquista s'annonce aussi par une tempête, voire par un Déluge, le changement d'ère se fait brusquement et s'accompagne d'une série d'images propres au topos apocalyptique : nuages noirs, déluge de pluie, raz-de-marée et tornades, inondations et destruction des villes, noyade des habitants. C'est ainsi qu'Irving décrit le prélude aux événements désastreux qui se sont achevés par l'effondrement du royaume mauresque de Grenade. 
Torrents came roaring down from the mountains, bringing with them rocks and trees ; the Darro overflowed its banks; mills were swept away ; bridges

destroyed, gardens laid waste. ${ }^{6} 6$

L'esthétique de la mort-mer est propre à l'imaginaire américain. Cooper également développe cette métaphore dans The Last of the Mohicans - la victoire de la Mort et l'élimination de la race semblent fatales (« Death was everywhere, and in his most terrific and disgusting aspects ${ }^{37}$ ), et le raz-de-marée de l'invasion fait couler une mer de sang: "The flow of blood might be linkened to the outbreaking of a torrent ». Le désir que les hommes éprouvent de tuer et de mourir s'élargit aux dimensions de l'univers, et l'eau en devient sanglante.

La deep sea marque un seuil sur lequel il est dangereux de s'aventurer, et qu'il est encore plus périlleux de franchir, ou de déplacer. Chez Irving, la mort est frontalière : les frontières, propices aux croisements culturels, sont également des lieux de confrontation que l'impossibilité linguistique et morale de communiquer rend mortellement dangereux. La rencontre de cultures différentes sur un même terrain provoque la bataille et la mort : un ordre est détruit, un autre point.

\section{Cérémonies funéraires, héritage culturel et réconciliation des nations}

Cependant, pour Irving, l'Andalousie est le terrain de la réconciliation entre les civilisations, et il montre de la sorte les cérémonies funéraires en l'honneur d'un roi ou du représentant d'un peuple comme un moment de réunion entre les nations. C'est que les rois maures ont emprunté aux rois chrétiens une certaine manière de traiter les morts. Dans The Alhambra, Irving raconte comment, après la mort de Ferdinand III de Castille, Alhamar envoya ses ambassadeurs pour exprimer ses condoléances au nouveau roi Alphonse X. C'est ainsi qu'une troupe de cavaliers maures entoura la bière royale pendant les cérémonies mortuaires. Ce témoignage de grand respect était répété à chaque anniversaire du roi Ferdinand. Réciproquement, le prince castillan Don Philippe, frère d'Alphonse X, assiste le premier roi de la dynastie nasride, Mohammed ben Nazar, dit Alhamar, le fondateur de l'Alhambra, dans ses derniers instants. La mort de ce dernier est soudaine, Alhamar meurt en quelques heures « vomiting blood and in violent convulsions $»^{38}$. Il est dit que le roi est embaumé, enfermé dans un cercueil d'argent et enterré au sein de l'Alhambra, dans un tombeau de marbre précieux, au milieu des lamentations sincères de ses proches sujets, qui le saluent comme leur père.

23 Il se dessine par ailleurs une forme de symétrie entre les morts. Plusieurs gouverneurs et plusieurs morts ont construit l'Alhambra, mais c'est par la faute de Boabdil que celle de Rodéric sera compensée : l'un et l'autre doivent abandonner Grenade parce qu'ils se sont rendus coupables d'un crime. Rodéric paie le prix du viol de Florinda, tandis que Boabdil cède à la jalousie et à l'orgueil blessé, et fait assassiner les Abencérages (« that illustrious line who were here perfidiously massacred » ${ }^{39}$ ) par son armée. La tuerie provoque un déséquilibre et le Royaume de Grenade devient très vulnérable aux assauts de l'armée catholique. Cet épisode sanglant a laissé des traces sur les murs de l'Alhambra, d'où ne s'effaceront jamais les lamentations des morts - car la puissance du mythe tient justement à ceci qu'il est indélébile.

Et, précisément, la fin de la Reconquista et la conquête des Amériques sont pour Irving indissociables : Christophe Colomb est présent à Grenade lors de la remise des clefs de la ville par Boabdil, «the last of the Moorish kings ${ }^{40}$ aux rois catholiques, et c'est là aussi que sera prise la décision qui aboutira à la " christianisation du monde ». Dans ses Spanish Voyages of Discovery, Irving décrit le départ des Colons, 
leur arrivée sur la Côte de la Paria, et il compare les mœurs des conquérants et celles des autochtones. Il mentionne la description de la vie des aborigènes du Nouveau Monde par Vespucci. Leur manière de traiter les morts témoigne de leur foi en une seconde vie physique : " Having deposited the corpse in a cavern or sepulcher, they placed a jar of water and a few eatables at its head, and then abandoned it without moan or lamentation $»^{4}$. Avant de l'abandonner, ils laissent des vivres auprès du défunt, pour le cas où il se réveillerait. Dans sa Description de la Louisiane, Louis Hennepin consacre d'ailleurs un chapitre à des rites de la mort quelque peu semblables : « les festins de mort sont lugubres et tristes [...] ; les parents du mort sont dans un grand silence, et font paraitre un visage abattu, pour émouvoir les conviés à compassion $»^{42}$. Mais ce qui compte surtout, c'est cette idée que répand Hennepin, que les Indiens croient que la vie physique continue après la mort. Hennepin raconte avoir entendu que les aborigènes craignent de mourir et de passer dans l'au-delà " parce qu'on y vit sans boire et sans manger, je n'y veux donc pas aller, disent-ils, parce que je veux manger. » C'est pourquoi ils l'accusent de mentir et opposent à ses discours théologiques l'objection suivante : « est-ce qu'on peut vivre sans manger ? " ${ }^{43}$. Quant à la manière d'ensevelir les morts, elle doit être pleine de magnificence : on colore le visage du défunt et on met dans le cercueil tout ce qui lui appartient, parce que « quand il sera aux pays des morts ou des esprits il aura besoin de tout cet équipage pour la chasse ${ }^{44}$.

\section{Le Native, le civilisé, la mort et le renouveau}

Tocqueville lui aussi confronte les Natives et les conquérants, le continent vierge et ceux qui allaient le façonner : il relate le mythe de l'Amérique, sa genèse, sa nature, sa géographie, qu'il lie directement à la Mort. L'émerveillement naiff des Européens devant la contrée exotique qu'ils découvraient n'était pas sans rapport avec l' « influence énervante qui attachait l'homme au présent et le rendait insouciant de l'avenir », et qui empêchait les conquérants de voir " la mort [qui] était cachée sous ce manteau brillant ${ }^{45}$. L'Amérique du Nord est représentée comme un locus terribilis : tout y est grave et solennel, et les eaux de l' " océan turbulent et brumeux [qui] envelopp[e] ses rivages ${ }^{46}$ sont mortelles.

Tocqueville insiste sur la coprésence de la mort et de la vie. La forêt, la nature sont hantées par la mort, la civilisation est construite sur les cadavres. Tocqueville compare les forêts dont l'homme s'est rendu maitre à ces paysages où la mort frappait sans relâche et où " personne ne se chargeait d'enlever les débris qu'elle avait faits. Ils s'accumulaient donc les uns sur les autres ». Avant que l'industrie ne s'approprie la nature, il semble qu'il n'y ait rien que la terre sans bornes mais infertile

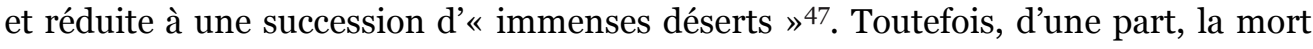
ouvre le chemin aux " jeunes rejetons » (ce qui est aussi l'idée de Jefferson ${ }^{48}$ ) : " Ainsi la mort venait en quelque sorte [...] aider à la vie. L'une et l'autre étaient en présence, elles semblaient avoir voulu mêler et confondre leurs œuvres ${ }^{49}$. Et surtout, ceux qui ont civilisé l'Amérique ont aussi tracé un sillon de mort, de telle manière que le pays nouveau s'est construit sur la mort de ses anciens habitants. L'industrialisation se développe donc au milieu des débris et de la mort. Le travail a fertilisé une terre inexploitée, mais c'est bien l'homme conquérant et industrialisant qui est responsable du meurtre systématisé, puisqu'il a créé une sorte de "machine à mort ». Cette idée revient d'ailleurs sous la plume de Cooper, qui déplore que la civilisation, qui n'est pour lui rien d'autre que la sœur de la monarchie, ait créé « an excess of pauperism, that hangs like a dead weigh on the nation ${ }^{50}$.

L'Industrialisation a donc renforcé le mythe romantique de l'Ancien Monde, du monde qui précède l'intervention destructrice de l'homme européen - mais même ce 
mythe préétabli des aborigènes contribue à faire reculer la wilderness. Le courage est glorifié dans l'imaginaire révolutionnaire et l'Indien semble être l'homme parfait parce qu'il est autonome : " l'Indien savait vivre sans besoins, souffrir sans se plaindre, et mourir en chantant $»^{51}$. Pourtant Tocqueville écrit que les Indiens attendaient la civilisation - c'est-à-dire leur propre mort, car les droits des découvreurs furent avant tout ceux des armes dont ils se servirent pour conquérir le pays, ce qu'Irving déplore et dénonce, notant que le seul droit des Indiens fut celui de mourir. La purification des terres et de leurs habitants par la foi chrétienne armée, par le « fire and sword, by stake and faggot ${ }^{52}$, fut si rapide qu'au bout de quelques années moins d'un cinquième des aborigènes étaient encore vivants.

La dialectique entre le Native et le civilisé, le traditionnel et l'industriel, se cristallise donc dans les représentations de la mort et dans le rapport à la mort : c'est par là qu'on distingue le sublime de l'ordinaire. La mort de l'individu est mise en scène et elle est élevée à la dignité d'une synecdoque : car la mort d'un homme est parfois la mort d'une culture, voire d'une civilisation. L'insoumission, le refus de céder devant l'ennemi rend la mort presque interminable, et la transforme en une véritable agonie - un combat contre l'ennemi et contre la Mort. L'imaginaire biblique du Jugement dernier est convoqué pour aborder les questions du gouvernement, de l'héritage, de la succession et de l'élimination, de telle façon que la mort physique du dernier homme est presque effacée au profit de la mort spirituelle de la civilisation. La Mort et sa violence sont représentées par le biais de la métaphore du raz-de-marée - image qui permet de supporter la violence, de l'atténuer pour pouvoir l'exprimer, en faisant apparaître la mort comme une catastrophe naturelle, dans laquelle l'homme ne peut et ne doit pas intervenir. Le conquérant efface ainsi sa responsabilité devant les morts, il se sert du prétexte biblique pour s'abstraire de la réalité physique du génocide : la catastrophe met fin à l'histoire, à une histoire, régénérant le monde et l'homme.

\section{Bibliographie}

Des DOI (Digital Object Identifier) sont automatiquement ajoutés aux références par Bilbo, l'outil d'annotation bibliographique d'OpenEdition.

Les utilisateurs des institutions abonnées à l'un des programmes freemium d'OpenEdition peuvent télécharger les références bibliographiques pour lesquelles Bilbo a trouvé un DOI.

Format

APA

MLA

Chicago

Le service d'export bibliographique est disponible pour les institutions qui ont souscrit à un des programmes freemium d'OpenEdition.

$\mathrm{Si}$ vous souhaitez que votre institution souscrive à l'un des programmes freemium d'OpenEdition et bénéficie de ses services, écrivez à : access@openedition.org.

Boussoulas, Nicolas-Isidore, La Peur et l'Univers dans l'œuvre d'Edgar Poe. Une Métaphysique de la peur, Paris : PUF, 1952.

Burnstein, Andrew et Isenberg, Nancy (éds.), Mortal Remains : Death in Early America, Philadelphia : University of Pennsylvania Press, 2003.

Cooper, James Fenimore, England : with Sketches of Society in the Metropolis, Paris : Baudry's European Library, 1837.

Cooper, James Fenimore, The Last of the Mohicans : A Narrative of 1757 [1826], New-York : G. P. Putman, 1850 .

Eliade, Mircea, Le Mythe de l'éternel retour. Archétypes et répétition, nouvelle édition revue et augmentée, Paris : Gallimard, « folio/essais », 1969.

Hennepin, Louis, Description de la Louisiane, Nouvellement découverte au Sud-Ouest de la Nouvelle France, Paris : Chez la Veuve Sebastien Huré, 1683.

Irving, Pierre M. (éd.), Life and Letters of Washington Irving, vol. 1, New-York : G. P. Putman, 1864 .

Irving, Pierre M. (éd.), Life and Letters of Washington Irving, vol. 3, London : Bentley, 1863. 
Irving, Washington, The Works of Washington Irving : Life and Voyages of Columbus [1828], revised edition, 3 vol., New-York: G. P. Putman, 1861.

Irving, Washington, The Alhambra [1832], author's revised edition, New-York : G. P. Putman, 1861.

Irving, Washington, Spanish Papers, edited by Pierre Monroe Irving, Philadelphia : J. B. Lippincott \& Co, 1872.

Irving, Washington, Spanish Voyages of Discovery, New-York : John W. Lovell Company, 1883 .

Mather, Cotton, Magnalia Christi Americana ; or, The Ecclesiastical History of New-England ; from its First Planting, in the Year 1620, unto the Year of our Lord 1698, 2 vol., Hartford : Silas Andrus \& Son, 1853.

Miller, Perry (éd.), The American Puritans. Their Prose and Poetry, New-York : Anchor Books, 1956.

Navarrete, Martín Fernández, Viages de Vespucio, in Colección de los viages y descubrimientos, que hicieron por mar los españoles desde fines del siglo $X V$, Tomo III, Madrid : En la Imprenta Real, 1829.

Punter, David, « Early American Gothic » in The Literature of Terror : a history of gothic fictions from 1765 to the present day. Volume 1. The Gothic tradition, London/New-York : Longman, (2 éd.), 1996.

Scott, Walter, The Vision of Roderick. A Poem, Boston : Greenleaf, 1811.

Southey, Robert, Roderick : The Last of the Goths [1814], 2 vol., London : Longman, Hurst, Rees, Orme, and Brown, 1816.

Thiesse, Anne-Marie, La Création des identités nationales. Europe XVIIIe - XXe siècle Paris : Seuil, coll. « L’Univers Historique », 1999.

Format

APA

MLA

Chicago

Le service d'export bibliographique est disponible pour les institutions qui ont souscrit à un des programmes freemium d'OpenEdition.

$\mathrm{Si}$ vous souhaitez que votre institution souscrive à l'un des programmes freemium d'OpenEdition et bénéficie de ses services, écrivez à : access@openedition.org.

Thiesse, Anne-Marie, «Des fictions créatrices : les identités nationales » in Romantisme, 2000, $\mathrm{n}^{\circ} 110$, p. 51-62.

DOI : $10.3406 /$ roman.2000.953

Tocqueville, Alexis De, De la Démocratie en Amérique [1835 et 1840], in Euvres complètes, publiées par Madame de Tocqueville, vol. 1, 15ème éd., Paris : Michel Lévy Frères, 1868.

\section{Notes}

1 Anne-Marie Thiesse écrit que c'est « au cours du XIXe siècle [que] s'est opérée la conversion de l'Europe des princes à l'Europe des nations. » Voir son article « Des fictions créatrices : les identités nationales » in Romantisme, 2000, $\mathrm{n}^{\circ} 110$, p. 51.

2 Thiesse, Anne-Marie, La Création des identités nationales. Europe XVIIIe-XXe siècle, Paris : Seuil, coll. «L'Univers Historique », 1999, p. 21.

3 Irving, Washington, « To the public », in A History of New-York, from the Beginning of the World to the End of the Dutch Dynasty, by Diedrich Knickerbocker, in Life and Works of Washington Irving, Richard Henry Stoddard (éd.), New-York : Pollard \& Moss, 188o, p. 545.

4 Ibid., p. 548.

5 Irving, Washington, « The Voyage » in The Sketch-Book of Geoffrey Crayon, Gent, in Life and Works of Washington Irving, p. 2.

6 Irving, Washington, "The Mutability of Literature ", in The Sketch-Book of Geoffrey Crayon, Gent, p. 33 .

7 Voir Punter, David, " Early American Gothic » in The Literature of Terror : a history of gothic fictions from 1765 to the present day. Volume 1. The Gothic tradition, London/New-York : Longman, (2 éd.), 1996, p. 165.

8 Irving, Washington, «Traits of Indian Character », in The Sketch-Book of Geoffrey Crayon, Gent, p. 72.

9 Idem. 
10 Ibid., p. 74.

11 Idem.

12 Ibid., p. 73.

13 Idem.

14 Ibid., p. 74.

15 Tocqueville, Alexis De, «De la Démocratie en Amérique » [1835 et 1840], in Euvres complètes, publiées par Madame de Tocqueville, vol. 1, Paris : Michel Lévy Frères, 1868, p. 36.

16 Irving, Washington, « Westminster Abbey », in The Sketch-Book of Geoffrey Crayon, Gent, p. 45 .

17 Irving, Washington, « The Legend of Sleepy Hollow, found among the papers of the late Diedrich Knickerbocker », in The Sketch-Book of Geoffrey Crayon, Gent, p. 90.

18 Burnstein, Andrew et Isenberg, Nancy (éds.), «Introduction » in Mortal Remains : Death in Early America, Philadelphia : University of Pennsylvania Press, 2003, p. 12.

19 Voir Irving, Pierre M. (éd.), Life and Letters of Washington Irving, vol. I, New York : G. P. Putman, 1864, p. 226.

20 Irving, Washington, The Alhambra [1832], author's revised edition, New-York : G. P. Putman, 1861, p. 120.

21 Idem.

22 Ibid., p. 252-253 : " I can convey the dead body out of the city, and bury it in the sands on the banks of the Xenil. No one saw the Moor enter our dwelling, and no one will know anything of his death. ».

23 Mather, Cotton, " Heralds of War » in Magnalia Christi Americana ; or, The Ecclesiastical History of New-England ; from its First Planting, in the Year 1620, unto the Year of our Lord 1698, 2 vol., Hartford : Silas Andrus \& Son, 1853, vol. 2, pp. 559-560.

24 Eliade, Mircea, Le Mythe de l'éternel retour. Archétypes et répétition, Paris : Gallimard, " folio/essais ", 1969, p. 139. Mircea Eliade parle de "l'éternelle répétition du rythme fondamental du Cosmos : sa destruction et sa re-création périodique », p. 134.

25 Irving, Washington, « The Legend of Don Roderick » in Spanish Papers, Philadelphia : J. B. Lippincott \& Co, 1872, pp. 82-83.

26 Scott, Walter, The Vision of Roderick. A Poem, Boston : Greenleaf, 1811.

27 Southey, Robert, Roderick : The Last of the Goths [1814], in 2 vol., vol. 1, London : Longman, Hurst, Rees, Orme, and Brown, 1816, p. ix : "These four persons [Witiza, Theodofred, Favila, the Wife of Favila] are dead before the action of the poem commences. »

28 Ibid., p. 38.

29 Ibid., pp. 1-2. (C'est une allusion aux sept plaies d'Égypte, Exode X, 3-7.)

30 Irving, Washington, The Alhambra, p. 68.

31 Irving, Washington, "Preface ", Legend of the Conquest of Spain, in Life and Works of Washington Irving, p. 295

32 Ibid., p. 299.

33 Irving, Washington, The Alhambra, p. 77.

34 Idem.

35 Boussoulas, Nicolas-Isidore, La Peur et l'Univers dans l'œuvre d'Edgar Poe. Une Métaphysique de la peur, Paris : PUF, 1952, p. 2.

36 Irving, Washington, The Alhambra, p. 168.

37 Cooper, James Fenimore, The Last of the Mohicans : A Narrative of 1757 [1826], New-York : Putman, 1850, p. 222.

38 Irving, Washington, The Alhambra, pp. 88-89.

39 Ibid., p. 56.

40 Irving, Washington, "Introduction ", in The Works of Washington Irving : Life and Voyages of Columbus [1828], revised edition in 3 vol., vol. 1, New-York : G. P. Putman, 1861, p. 110.

41 Irving, Washington, "Customs of the Nations ", in Spanish Voyages of Discovery, New-York : John W. Lovell Company, 1883, p. 13.

Irving se réfère à l'ouvrage de Martín Fernández Navarrete, Viages de Vespucio, in Colección de los viages y descubrimientos, que hicieron por mar los españoles desde fines del siglo $X V$, Tomo III, Madrid : En la Imprenta Real, 1829, pp. 221-222. 
42 Hennepin, Louis, « Les Mœurs et la manière de vivre des Sauvages ", in Description de la Louisiane, Nouvellement découverte au Sud-Ouest de la Nouvelle France, Partie II, Paris : Chez la Veuve Sebastien Huré, 1683, p. 45.

43 Ibid., p. 95.

44 Ibid., pp. 90-91.

45 Tocqueville, Alexis De, « Configuration de l'Amérique du Nord » in De la Démocratie en Amérique, p. 32.

46 Idem.

47 Ibid., p. 33.

48 Dans une lettre adressée à James Madison, datée du 6 septembre 1789, Jefferson écrit : « the earth belongs in usufruct to the living : that the dead have neither powers nor rights over it ».

49 Tocqueville, Alexis De, «Configuration de l'Amérique du Nord » in De la Démocratie en Amérique, p. 33.

50 Cooper, James Fenimore, England : with Sketches of Society in the Metropolis, Paris : Baudry's European Library, 1837, p. 127.

51 Tocqueville, Alexis De, De la Démocratie en Amérique, p. 37.

52 Irving, Washington, A History of New-York, p. 554.

\section{Pour citer cet article}

Référence électronique

Nikol Dziub, «L'imaginaire transatlantique de la mort : rites, mythes et conflits », Amerika [En ligne], 12 | 2015, mis en ligne le 03 juillet 2015, consulté le 08 décembre 2015. URL :

http://amerika.revues.org/6201; DOI : 10.4000/amerika.6201

\section{Auteur}

Nikol Dziub

Université de Haute-Alsace

\section{Droits d'auteur}

(C) Tous droits réservés 